\title{
Topic Models for Assessment of Mental Health Issues
}

\author{
Maxime D. Armstrong, Diego Maupomé and Marie-Jean Meurs* \\ Université du Québec à Montréal, Montréal, QC, Canada
}

\begin{abstract}
In this paper, we explore topic modeling for the assessment of risk for depression, anorexia and self-harm. Using social media textual content from different datasets, we focus on Latent Dirichlet Allocation models, trained on both specific and combined corpora made from these datasets to perform risk detection. We investigate mental health vocabulary and shared topic modeling performance improvements on user classification.
\end{abstract}

Keywords: Topic modeling · Risk assessment - Social media · Sentiment analysis . Natural language processing

\section{Introduction}

On a global scale, mental health issues account for a significant portion of the total burden of disease, up to $13 \%$ according to recent studies [1]. For instance, in North America in 2018, $18 \%$ of Canadians seeking health care did so in relation to mental health, making it the most common reason to request for care; the proportion being higher within those aged 15 to 34, reaching almost $60 \%$ [2]. Among mental health issues, depression is one of the most common. In Europe, depression is a highly prevalent condition, with an estimated 33.4 million people affected [3]. In the U.S., depression reaches an annual prevalence rate of $7.8 \%$ among adults, which represent a total of 19.4 million people affected every year [4]. According to a recent study [5], the prevalence rate of depression among the adult population in China is estimated as high as $38 \%$.

Mental health issues also cover eating disorders, such as anorexia, which reach the highest overall mortality rate of any mental illness, with estimates between 10-15\% [6]; the mortality rate associated with anorexia peaks for females aged 15-24 years old, being 12 times greater than that all other causes of death combined [7]. Self-harm is also an important issue, particularly among youth. In Canada, which is among the few countries where very detailed statistics are available, in 2013-2014, about 2,500 hospitalizations among youth age 10 to 17 were due to intentional self-harm, representing 1 in 4 injury hospitalizations for youth in this age group [8]. Since early intervention on mental health issues gives better treatment results $[9,10]$, this work focuses on early assessment of risk for depression, anorexia and selfharm with topic modeling and automatic detection from text extracted from social media. The long-term goal is to help mental health practitioners acquire better tools to assess the mental status of patients using AI-based systems. This could save precious time in the diagnostic and treatment processes.

Topic modeling has shown promising results for mental health issue detection. Resnik, Armstrong, Claudino, Nguyen, Nguyen, and Boyd-Graber [11] demonstrated that topic models such as Latent Dirichlet Allocation (LDA) [12] can uncover meaningful and promising latent structure within depression-related language collected from Twitter. Coppersmith, Dredze, Harman, Hollingshead, and Mitchell [13] confirmed the potential of using social media content such as Twitter posts for depression and Post-Traumatic Stress Disorder (PTSD) binary classification. In addition, Zhao, Jiang, Weng, He, Lim, Yan, and Li [14] showed the potential of using Twitter content for topic extraction, and Jelodar, Wang, Yuan, Feng, Jiang, Li, and Zhao [15] reiterate on topic modeling and LDA capacities to

*meurs.marie-jean@uqam.ca

This article is (C) 2021 by author(s) as listed above. The article is licensed under a Creative Commons Attribution (CC BY 4.0) International license (https://creativecommons.org/licenses/by/4.0/legalcode), except where otherwise indicated with respect to particular material included in the article. The article should be attributed to the author(s) identified above. 
Table 1. Summary statistics of the datasets from the eRisk Shared Tasks.

\begin{tabular}{|l|r|r||r|r||r|r|}
\cline { 2 - 7 } \multicolumn{1}{c|}{} & \multicolumn{2}{c|}{ Depression (2018 T1) } & \multicolumn{2}{c||}{ Anorexia (2019 T1) } & \multicolumn{2}{c|}{ Self-harm (2020 T1) } \\
\cline { 2 - 7 } \multicolumn{1}{c|}{} & \multicolumn{1}{c|}{ Risk } & Control & \multicolumn{1}{c|}{ Risk } & Control & \multicolumn{1}{c|}{ Risk } & \multicolumn{1}{c|}{ Control } \\
\hline Nb. users & 214 & 1493 & 134 & 1153 & 145 & 618 \\
\hline Nb. writings & 90222 & 986360 & 42493 & 781768 & 18987 & 255964 \\
\hline \hline writings / user & 411.6 & 660.7 & 317.1 & 678.0 & 130.9 & 414.2 \\
\hline avg. writing length (words) & 27.5 & 22.8 & 37.3 & 21.5 & 31.5 & 21.5 \\
\hline
\end{tabular}

uncover hidden structures related to user behavior in social media. Although previous work demonstrates the effectiveness of topic modeling for depressive-related conditions, there is a lack of horizontal application of this approach to various mental health issues, so as to compare them and assess its potential on a case-by-case basis. Furthermore, combining corpus concerning different mental health issues to perform such approach does not appear to have been tested yet, to the best of our knowledge.

The main contributions of this work are put forward on two different levels. Primarily, we perform binary classification with different specific topic models to observe their potential, and evaluate whether topic models related to mental health assessment showing a distinctive and strong vocabulary achieve better results. Moreover, we explore how shared topic models might improve risk detection of mental health issues sharing similar lexical field. Specifically, we study whether training a single topic model over corpora pertaining to different mental health issues benefits the assessment of such issues.

The rest of the paper is organized as follows. Section 2 gives a brief overview of the use of topic models for mental health assessment, introduces the data used and our approach. Section 3 presents our experimental settings and the results obtained, while Section 4 analyzes them. Finally, Section 5 proposes future work to be done.

\section{Topic Detection on User-generated Textual Content}

Over recent years, social media textual content has been shown to be an interesting basis of discovery of the mental status of its authors $[16,17]$. Social media users might share their feelings and circumstances with one another. As such, the topics discussed on Internet fora can help in detecting at-risk persons $[11,18]$. By extracting topics from data concerning different mental health issues, this work aims to study how robust LDA is at separating the topics concerning each one. To that end, the experiments evaluate the impact of both shared and specific topic modeling. While specific topic modeling consists in extracting topics from a dataset associated to a sole mental health issue, shared topic modeling is performed on combined corpora, i.e. the combination of specific datasets into a single corpus. The data used are borrowed from the recurring eRisk shared task, broadly aimed at assessing the risk for different mental health issues from social media text.

Data. The data consist of three distinct datasets pertaining to depression [19], anorexia [20] and self-harm [21]. Each dataset is composed of the written production of English-speaking Reddit users. Each dataset contains writings from two classes of users. Risk users (i.e. positive users) have admitted to having been diagnosed with a mental health issue; control users (i.e. negative users) have not. Table 1 presents the number of users and writings for each class in each dataset. For positive users, the text content extracted precedes and excludes the mention of diagnosis. All three datasets were originally split into a training set and a test set. However, these partitions show large discrepancies in class proportions and subject verbosity. For this reason, we perform instead five-fold cross-validation on the complete datasets for each task.

Model. The topics discussed in the data are extracted using the well-known LDA algorithm [12]. The per-user distribution of topics is then used to predict the risk for the 
relevant mental health issue. LDA posits documents as having a topic distribution sampled from a Dirichlet prior, each topic having its own distribution over words, also sampled from a Dirichlet distribution. The observation of each word in a document is therefore modeled as the result of sampling a topic from the distribution of $k$ topics assigned to said document and sampling the word from this topic distribution. As $k$ fluctuates, LDA evaluates different distributions of topics to best fit all the observed documents. By inferring an LDA model, and thus the unobserved topics that resulted in the set of observed documents, LDA allows to discover the underlying structure within a collection of documents. Having fit an LDA model across the selected documents, one can then represent a user's writings as a proportion of topics. These proportions allow to map the user's writings to a prediction of the user's risk.

\section{Experiments and Results}

Preprocessing. A training corpus $C$ is built from one or more of the eRisk datasets previously described. In this case, four corpora were created for further model training. The first three corpora consist of the three eRisk datasets as is, denoted as $C_{D}$ for depression, $C_{A}$ for anorexia and $C_{S}$ for self-harm. To test shared topic modeling, the depression and selfharm datasets were combined into a sole corpus, $C_{D S}$, since both datasets share a similar lexical field, while the anorexia one does not. The documents from the original datasets were simply put together to create a larger corpus.

The LDA models were trained on documents obtained by preprocessing the corpora. Each user of a dataset is considered as a document in a corpus i.e. the user's writings are concatenated to generate a sole document. Stop words and short words (3 characters or fewer) are removed in every document. Following [22], the remaining words are stemmed and extremes are filtered before training, i.e. words appearing in less than 20 documents or more than $50 \%$ of the documents are removed.

Training. Four different LDA models were tested, one for each of the training corpora. According to the nomenclature of the corpora, they are respectively denoted $L D A_{D}$, $L D A_{A}, L D A_{S}$ and $L D A_{D S}$. To improve the external validity of the findings, five-fold crossvalidation is performed. Each model and ensuing classifiers are trained on $80 \%$ of users and evaluated on the remaining $20 \%$, reported results being the mean across the folds. Once an LDA model is trained, users are mapped to a vector of topics. Finally, four binary classifiers are trained on these vectors - using Logistic Regression (LR), $k$-Nearest Neighbors (KNN), Multi-Layer Perceptron (MLP) and Random Forests (RF). Note that for $L D A_{D S}$, two sets of classifiers are created, one for depression and one for self-harm. Thus, only vectors resulting from users' productions belonging to a specific dataset are exploited to train the classifiers and test the prediction of a mental health issue. This ensures that the original labels are respected, without adding "noisy" users from another dataset during the binary classification. Different training-validation splits are used to tune the hyper-parameters, namely the number of topics extracted. The number of topics varies from 10 to 30, as such range showed promising results for the assessment of mental health issues [18, 22].

Evaluation. F-measure $\left(\mathrm{F}_{m}\right)$ is used as the main metric to evaluate the results. $\mathrm{F}_{m}$ is the harmonic mean of precision $(\mathrm{P})$ and recall $(\mathrm{R})$ of the positive class, as it is the most difficult one to assess for the task. This allows to balance a retrieval of positive observations that is both exhaustive and discerning. For each corpus, the number of topics achieving the best $\mathrm{F}_{m}$ is reported.

Results. The results for the best model for each corpus are presented in Table 2 where $L D A_{C} @ k$ denotes the LDA model with $k$ topics trained on corpus $C$. Overall, the best results were obtained for the classification of anorexia, followed by self-harm then depression. 
Table 2. Precision $(\mathrm{P})$, recall $(\mathrm{R})$ and $\mathrm{F}$-measure $\left(\mathrm{F}_{m}\right)$ for depression, self-harm and anorexia for LDA models.

\begin{tabular}{|c|c|c|c|c|c|c|c|c|c|c|c|c|c|}
\hline & \multirow[b]{3}{*}{ Model } & \multicolumn{12}{|c|}{ Classifier } \\
\hline & & \multicolumn{9}{|c|}{\begin{tabular}{l}
\multicolumn{2}{c}{ Classifier } \\
\end{tabular}} & & & \\
\hline & & \multicolumn{3}{|c|}{$\frac{116}{\mathrm{R}}$} & $\mathrm{P}$ & $\mathrm{R}$ & $\mathrm{F}_{m}$ & & & & $\mathrm{P}$ & $\mathrm{R}$ & $\mathrm{F}_{m}$ \\
\hline \multirow[t]{2}{*}{ Depression } & $L D A_{D} @ 25$ & 0.44 & 0.77 & 0.56 & 0.66 & 0.43 & 0.52 & 0.63 & 0.47 & 0.53 & 0.77 & 0.29 & 0.41 \\
\hline & $L D A_{D S} @ 25$ & 0.51 & 0.78 & 0.61 & 0.65 & 0.45 & 0.52 & 0.64 & 0.52 & 0.57 & 0.74 & 0.33 & 0.45 \\
\hline \multirow[t]{2}{*}{ Self-harm } & $L D A_{S} @ 19$ & 0.60 & 0.76 & 0.67 & 0.71 & 0.61 & 0.65 & 0.80 & 0.59 & 0.68 & 0.86 & 0.40 & 0.54 \\
\hline & $L D A_{D S} @ 25$ & 0.20 & 0.53 & 0.29 & 0.26 & 0.08 & 0.12 & 0.29 & 0.02 & 0.04 & 0.10 & 0.01 & 0.01 \\
\hline Anorexia & $L D A_{A} @ 18$ & 0.71 & 0.87 & 0.78 & 0.89 & 0.70 & 0.78 & 0.88 & 0.72 & 0.79 & 0.88 & 0.58 & 0.70 \\
\hline
\end{tabular}

Table 3. Vocabulary of the most evocative mental health topic for depression, anorexia and self-harm.

\begin{tabular}{|l|l|}
\cline { 2 - 2 } \multicolumn{1}{c|}{} & Top 10 stems (descending order of probability) \\
\hline$L D A_{D S} @ 25$ & depress-, women, relationship, doctor, pain, medic-, anxieti-, mental, okay, husband \\
\hline$L D A_{A} @ 18$ & weight, calori-, disord-, healthi-, doctor, gain, bing, relationship, diet, depress- \\
\hline$L D A_{S} @ 19$ & parent, famili-, depress, pain, women, relationship, self, mental, felt, situat- \\
\hline
\end{tabular}

For depression, $L D A_{D S} @ 25$ reached a maximal $\mathrm{F}_{m}$ of 0.61 with an LR classifier. For anorexia, $L D A_{A} @ 18$ hit the highest $\mathrm{F}_{m}$ of 0.79 with an MLP classifier. For self-harm, $L D A_{S} @ 19$ got to a $\mathrm{F}_{m}$ of 0.68 with a MLP classifier. In addition, for the three best models, the topic with the most evocative mental health vocabulary was extracted from the generated topics. The first 10 stems - showing the highest probability within the topic - are displayed in Table 3, in descending order of probability.

\section{Discussion}

The results obtained uphold our initial hypotheses. Some mental health issues appear to show strong and distinctive vocabulary, such as the examples of depression and anorexia presented in Table 3, which allows good performance in classification with topic modeling. Using larger datasets for anorexia and depression resulted in human readable topics, contrary to self-harm. The top 10 stems for the most evocative topic for $L D A_{A} @ 18$ express significant vocabulary, and appear to be highly related to anorexia, while those for $L D A_{S} @ 19$ seem less distinctive to self-harm, including stems like parent, famili- and relationship. Similarly, the $L D A_{D S} @ 25$ top stems appear also less distinctive than the $L D A_{A} @ 18$ ones despite the corpora size, including stems like women, relationship and okay.

In addition, the model trained with combined datasets, which presented similar lexical content, appears to be more effective in some cases. The experiments showed that $L D A_{D S}$ gave better results for depression classification than $L D A_{D}$, suggesting that extending corpus with similar mental health-related lexical content could help improve classification. However, tests also demonstrate that $L D A_{D S}$ underperformed for self-harm classification. This may be due to the imbalance between the depression and self-harm datasets, diluting self-harm-specific vocabulary and resulting in weaker classification performance.

It should be noted that the self-harm dataset spans a vocabulary much smaller than the other datasets, even after stemming. At $96 \mathrm{k}$ word types before preprocessing, the vocabulary is less than half the size of the vocabularies of the depression set (274k word types) and the anorexia set (238k word types). This also stands for word occurrences, since the selfharm dataset neighbours $6 \mathrm{M}$ words, while their counts is much higher for the depression set $(25 \mathrm{M})$ and the anorexia set $(18 \mathrm{M})$. Furthermore, the vocabulary usage seems less spread out in the self-harm dataset. Combining the depression and self-harm datasets results in a vocabulary with a distribution much more similar to that of the depression dataset. This is illustrated in Figure 1. The systems shown better results in comparison to those obtained during the eRisk competition as reported in Table 4. It is to be noted though that the splits used for this task were slightly different from those in the previous eRisk shared tasks. 


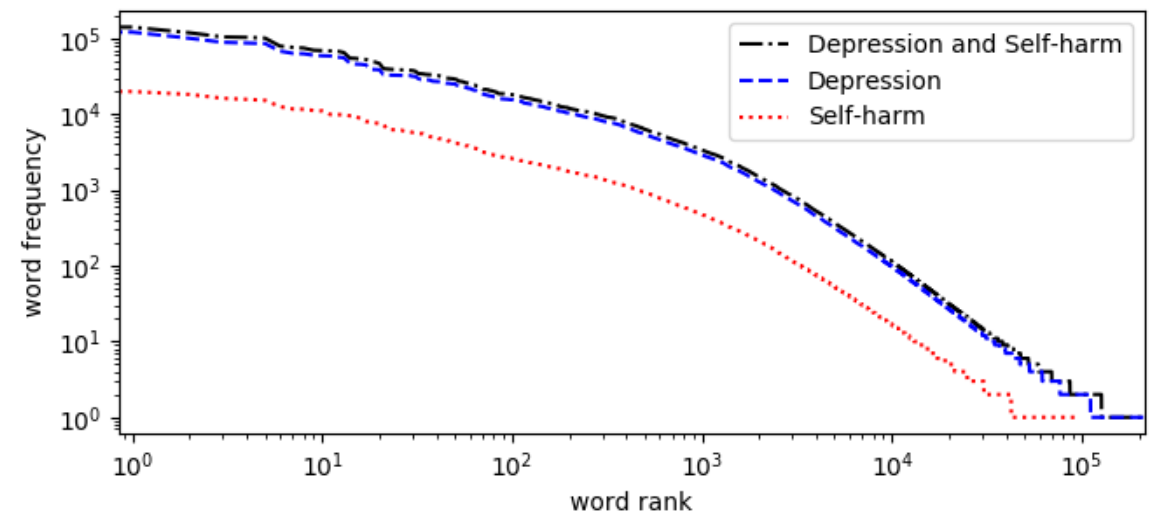

Figure 1. Distribution of frequency to rank in the vocabularies of the Depression and Self-harm datasets as well as the combined dataset. Frequency is in total number of occurrences. Both axes are in logarithmic scale.

Table 4. Comparative F-measure $\left(\mathrm{F}_{m}\right)$ results between eRisk shared tasks and our best models. TM and $\mathrm{BM}$ refer respectively to topic model and best model.

\begin{tabular}{|l|l|l|}
\cline { 2 - 3 } \multicolumn{1}{c|}{} & Model & $\mathrm{F}_{m}$ \\
\hline Depression & eRisk 2018 TM & 0.42 \\
\cline { 2 - 3 } & $L D A_{D} @ 25$ & 0.56 \\
\cline { 2 - 3 } & $L D A_{D S} @ 25$ & 0.61 \\
\hline Anorexia & eRisk 2019 BM & 0.71 \\
\cline { 2 - 3 } & $L D A_{A} @ 19$ & 0.79 \\
\hline
\end{tabular}

Both $L D A_{D}$ and $L D A_{D S}$ models score better results for depression classification than the team proposing a topic model for eRisk2018 T1 [19], which obtained a $\mathrm{F}_{m}$ of 0.42 at the time. With a $\mathrm{F}_{m}$ of 0.79 , the proposed $L D A_{A}$ model surpasses every model proposed for eRisk2019 T1 [20], for which the best $\mathrm{F}_{m}$ was 0.71 .

\section{Conclusion and Future Work}

Exploring both shared and specific mental health-related topic models, various LDA models were tested to assess automatic detection of depression, anorexia and self-harm. The most evocative topic was extracted for each of the best models to investigate vocabulary distinctiveness, and analyze the classification performance. The reported results confirm the potential of topic modeling for early assessment of mental health issues with distinctive vocabulary. Future work could include training LDA models on combined corpora made from balanced datasets. Also, diverse topic models could be tested with the same corpora, such as Supervised LDA and Supervised Anchor, which showed promising results for depression-related language [11]. In addition, an online LDA with infinite vocabulary approach as suggested by [23] could be interesting for topic models trained on users written online contributions in social media.

Reproducibility. The source code of the proposed systems is licensed under the GNU GPLv3. The datasets are provided on demand by the eRisk organizers.

\section{Acknowledgments}

This research was enabled in part by support provided by Calcul Québec and Compute Canada. MJM acknowledges the support of the Natural Sciences and Engineering Research Council of Canada [NSERC Grant number 06487-2017] and the Government of Canada's New Frontiers in Research Fund (NFRF), [NFRFE-2018-00484]. 


\section{References}

[1] World Health Organization. Mental Health Action Plan 2013-2020. World Health Organization, 2013.

[2] Statistics Canada. Care Counts: Receiving Care for a Mental Illness, 2018. 2020.

[3] World Health Organization. Preventing Depression in the WHO European Region. World Health Organization, 2016.

[4] Substance Abuse and Mental Health Services Administration. Key Substance Use and Mental Health Indicators in the United States: Results from the 2019 National Survey on Drug Use and Health. 2020.

[5] X. Qin, S. Wang, and C.-R. Hsieh. "The Prevalence of Depression and Depressive Symptoms among Adults in China: estimation based on a National Household Survey". In: China Economic Review (2018).

[6] J. Arcelus, A. J. Mitchell, J. Wales, and S. Nielsen. "Mortality Rates in Patients with Anorexia Nervosa and other Eating Disorders: A Meta-Analysis of 36 Studies". In: Archives of General Psychiatry, 68. 2011.

[7] F. E. Smink, D. van Hoeken, and H. W. Hoek. "Epidemiology of Eating Disorders: Incidence, Prevalence and Mortality Rates". In: Current Psychiatry Reports 14. 2012.

[8] Canadian Institute for Health Information. Intentional Self-Harm among Youth in Canada. 2014.

[9] C. Arango, C. M. Díaz-Caneja, P. D. McGorry, J. Rapoport, I. E. Sommer, J. A. Vorstman, D. McDaid, O. Marín, E. Serrano-Drozdowskyj, R. Freedman, et al. "Preventive Strategies for Mental Health". In: The Lancet Psychiatry (2018).

[10] Canadian Mental Health Association. Early Intervention. 2020.

[11] P. Resnik, W. Armstrong, L. Claudino, T. Nguyen, V.-A. Nguyen, and J. Boyd-Graber. "Beyond LDA: Exploring Supervised Topic Modeling for Depression-Related Language in Twitter". In: Workshop on Computational Linguistics and Clinical Psychology. 2015.

[12] M. I. J. David M. Blei Andrew Y. Ng. "Latent Dirichlet Allocation". In: Journal of Machine Learning Research 3. 2003, pp. 993-1022.

[13] G. Coppersmith, M. Dredze, C. Harman, K. Hollingshead, and M. Mitchell. "CLPsych 2015 Shared Task: Depression and PTSD on Twitter". In: Workshop on Computational Linguistics and Clinical Psychology. 2015.

[14] W. X. Zhao, J. Jiang, J. Weng, J. He, E.-P. Lim, H. Yan, and X. Li. "Comparing Twitter and Traditional Media using Topic Models". In: Advances in Information Retrieval. 2011.

[15] H. Jelodar, Y. Wang, C. Yuan, X. Feng, X. Jiang, Y. Li, and L. Zhao. "Latent Dirichlet Allocation (LDA) and Topic Modeling: Models, Applications, a Survey". In: Multimedia Tools and Applications (2019).

[16] J. Ive, G. Gkotsis, R. Dutta, R. Stewart, and S. Velupillai. "Hierarchical Neural Model with Attention Mechanisms for the Classification of Social Media Text Related to Mental Health". In: Workshop on Computational Linguistics and Clinical Psychology. 2018.

[17] R. M. Merchant, D. A. Asch, P. Crutchley, L. H. Ungar, S. C. Guntuku, J. C. Eichstaedt, S. Hill, K. Padrez, R. J. Smith, and H. A. Schwartz. "Evaluating the Predictability of Medical Conditions from Social Media Posts". In: PLOS ONE (2019).

[18] D. Maupomé and M.-J. Meurs. "Using Topic Extraction on Social Media Content for the Early Detection of Depression." In: CLEF (Working Notes) 2125 (2018).

[19] D. E. Losada, F. Crestani, and J. Parapar. "Overview of eRisk 2018: Early Risk Prediction on the Internet". In: CLEF (Working Notes). 2018.

[20] D. E. Losada, F. Crestani, and J. Parapar. "Overview of eRisk 2019 Early Risk Prediction on the Internet". In: International Conference of the Cross-Language Evaluation Forum for European Languages. Springer. 2019.

[21] D. E. Losada, F. Crestani, and J. Parapar. "Overview of eRisk 2020: Early Risk Prediction on the Internet". In: Experimental IR Meets Multilinguality, Multimodality, and Interaction International Conference of the CLEF Association (CLEF 2020). Ed. by A. Arampatzis, E. Kanoulas, T. Tsikrika, S. Vrochidis, H. Joho, C. Lioma, C. Eickhoff, A. Névéol, L. Cappellato, N. Ferro (eds). Springer International Publishing, 2020.

[22] D. Maupomé, M. D. Armstrong, R. Belbahar, J. Alezot, R. Balassiano, M. Queudot, S. Mosser, and M.-J. Meurs. "Early Mental Health Risk Assessment through Writing Styles, Topics and Neural Models". In: CLEF (Working Notes). 2020.

[23] K. Zhai and J. Boyd-Graber. "Online Latent Dirichlet Allocation with Infinite Vocabulary". In: International Conference on Machine Learning. 2013. 